\title{
A Geometric Inequality and the Complexity of Computing Volume
}

\author{
G. Elekes
}

Mathematical Institute, Eötvös Loránd University, H-1088 Budapest, Muzeum krt. 6-8, Hungary

Dedicated to my teacher Kö̀váry Károly

\begin{abstract}
The volume of the convex hull of any $m$ points of an $n$-dimensional ball with volume $V$ is at most $V \cdot m / 2^{n}$. This implies that no polynomial time algorithm can compute the volume of a convex set (given by an oracle) with less than exponential relative error. A lower bound on the complexity of computing width can also be deduced.
\end{abstract}

\section{An Extremal Problem in $n$-Dimensional Geometry}

In this section we deal with the following question:

Let $S$ be a ball in the $n$-dimensional Euclidean space with volume 1 . Choose any $m$ points $P_{1}, P_{2}, \ldots, P_{m} \in S$. Denote by $C_{m}$ the convex hull of $\left\{P_{i} ; 1 \leq i \leq m\right\}$. What is the maximum $v(n, m)$ of the volume $C_{m}$ maximized over all possible set of points?

Here we prove an upper bound on $v(n, m)$. This seems to be far from being tight (see Section 4); however, exponential lower bounds can be derived from it for some algorithmic problems. The latter will be discussed in Sections 2 and 3.

Theorem 1. $v(n, m) \leq m / 2^{n}$.

Proof. Let $O$ be the center of $S$. Denote by $S_{i}(1 \leq i \leq m)$ the ball with diameter $O P_{i}$. Naturally all vol $\left(S_{i}\right) \leq 1 / 2^{n}$.

Claim. $C_{m} \subseteq \bigcup\left\{S_{i} ; 1 \leq i \leq m\right\}$.

(This clearly implies the Theorem.)

Suppose that this claim is false, i.e., there is a point $Q$ of $C_{m}$ which is not contained in any $S_{i}$. This is equivalent to the property that $\Varangle O Q P_{i}<\pi / 2$ for all $1 \leq i \leq m$. 
Consider the hyperplane $H$ orthogonal to $O Q$ and going through $Q .{ }_{4} O Q P_{i}<$ $\pi / 2$ implies that for all $1 \leq i \leq m, P_{i}$ is in the same open halfspace determined by $H$ as $O$. Hence $Q$, which is on $H$, cannot be in the convex hull, a contradiction.

\section{The Complexity of Computing the Volume of an n-Dimensional Convex Body}

Grötschel et al. ([GLS], see also [L]) described a well-guaranteed separation oracle encoding a bounded convex subset $K$ of the $n$-dimensional space. This oracle works as follows:

(i) For any point $P$ it tells either that $P \in K$ or gives a hyperplane which separates $P$ from $K$.

(ii) It gives in advance a ball $S$ containing $K$ and another ball $S^{\prime}$ contained in $K$.

(Note that our oracle gives the centre of $S^{\prime}$, while that of [GLS] gives only its radius; but these two versions are polynomial time equivalent.)

They have shown that this oracle is polynomial time equivalent to a number of others, e.g., using this, one can minimize any linear function over $K$ in polynomial time. They have also proved that the volume of $K$ can be estimated up to a factor of size $n^{3 n / 2}$.

Here we show that this factor cannot be reduced to less than an exponential one.

Theorem 2. Suppose that an algorithm has access to a well-guaranteed separation oracle encoding $K$. If for some $c=c(n)<1$ the algorithm can give an estimate $v_{0}$ for $\operatorname{vol}(K)$ up to a factor $c$, i.e.,

$$
c \cdot v_{0} \leq \operatorname{vol}(K) \leq v_{0} \text {, }
$$

then its running time is at least $c \cdot 2^{n}-(n+1)$.

Proof. Suppose the oracle answers "yes" iff $P \in S$ and shows a hyperplane separating $\boldsymbol{P}$ from $S$ otherwise. Suppose, moreover, that it gives the vertices of a regular simplex inscribed in $S$ to be in $K$. (Without being asked for these points.) Note that this yields an inscribed ball $S^{\prime}$ of $K$.

If the algorithm asks fewer than $c \cdot 2^{n}-(n+1)$ other points then it will "know" only $m<c \cdot 2^{n}$ points $P_{1}, \ldots, P_{m}$ to be in $K$. Their convex hull $C_{m}$ will have

$$
\operatorname{vol}\left(C_{m}\right) \leq \operatorname{vol}(S) \cdot m / 2^{n}<\operatorname{vol}(S) \cdot c
$$

by Theorem 1 . So the algorithm cannot conclude that $K$-which may still be as large as $S$ itself or as small as $C_{m}$-has volume either at least $c \cdot \operatorname{vol}(S)$ or less than $\operatorname{vol}(S)$. 
Corollary 1. If an algorithm has access to a well-guaranteed separation oracle and can compute the volume of $K$ up to a factor $(2-\varepsilon)^{n}$ then its running time is exponential.

Proof. From Theorem 2 we have for $c=(2-\varepsilon)^{-n}$ that the running time is at least $[2 /(2-\varepsilon)]^{n}-(n+1)$.

\section{The Complexity of Computing Width}

The width of a convex body can be computed up to a factor $n^{3 / 2}$ in polynomial time ([GLS]). It can be derived from Theorem 1 that this factor cannot be better than 2.

Theorem 3. Suppose that an algorithm has access to a well-guaranteed separation oracle encoding $K$. If for some $\frac{1}{2}<d(n)<1$ the algorithm can give an estimate $w_{0}$ of the width of $K$ such that

$$
d \cdot w_{0} \leq \text { width }(K) \leq w_{0},
$$

then its running time is at least $\frac{1}{2}(2 d)^{n}-(n+1)$.

Proof. Suppose the oracle generously informs us that $K$ is symmetric with respect to the centre $O$ of $S$ and it works as described in the proof of Theorem 2 otherwise. If the algorithm asks fewer than $\frac{1}{2}(2 d)^{n}-(n+1)$ points then it will know only $m<(2 d)^{n}$ points of $K$ (the vertices of the regular simplex, the points it asked, and their image with respect to $O$ ). Denote the convex hull of all these points by $C_{m}$.

It is well known that a centrally symmetric convex body with width $w$ contains a ball of diameter $w$. Apply this for $C_{m}$ and denote $\operatorname{diam}(B) / \operatorname{diam}(S)$ by $\lambda$.

By Theorem 1 we have

$$
\operatorname{vol}(S) \cdot \lambda^{n}=\operatorname{vol}(B) \leq \operatorname{vol}\left(C_{m}\right)<\operatorname{vol}(S) \cdot d^{n}
$$

which means that $\lambda<d$, i.e., the algorithm cannot give an estimate with relative error at most $1 / d$.

\section{Open Problems}

It seems likely that for polynomially bounded $m=m(n), v(n, m)$ is superexponentially small, though this question is still open:

Problem. Is it true that for all $k \in \mathbb{N}$

$$
\left[v\left(n, n^{k}\right)\right]^{1 / n} \rightarrow 0 ?
$$


An affirmative answer to this would yield better lower bounds for the factors in the corollary. ( $\varepsilon^{n}$ (resp. $\varepsilon$ ) for all $\varepsilon<1$ (resp. 2) in Corollary 1.) ${ }^{1}$

Let us note that the problem of computing the volume of a convex polyhedron given either by a system of inequalities or by the set of its vertices in polynomial time still remains unsolved.

\section{Acknowledgment}

I wish to express my thanks to L. Lovász for calling my attention to the algorithmic problems, for suggesting simplifications to the proof of Theorem 1, and for his comments on the first version of the manuscript.

\section{References}

[GLS] M. Grötschel, L. Lovász, and A. Schrijver, Geometric methods in combinatorial optimization, in Progress in Combinatorial Optimization, Vol. 1 (W. R. Pulleyblank, ed.), 167-183, Academic Press, New York, 1984.

[L] L. Lovász, An Algorithmic Theory of Numbers, Graphs, and Convexity, AMS-SIAM Regional Conference Series, to appear.

Received November 21, 1985.

\footnotetext{
'I. Bárány and $Z$. Füredi have answered this problem affirmatively.
} 